\title{
THE NONEQUIVARIANT COHERENT-CONSTRUCTIBLE CORRESPONDENCE FOR TORIC SURFACES
}

\author{
TATSUKI KUWAGAKI
}

\begin{abstract}
We prove the nonequivariant coherent-constructible correspondence conjectured by Fang-Liu-Treumann-Zaslow in the case of toric surfaces. Our proof is based on describing a semi-orthogonal decomposition of the constructible side under toric point blow-up and comparing it with Orlov's theorem.
\end{abstract}

\section{Introduction}

The nonequivariant coherent-constructible correspondence (NCCC) is a relation between the derived category of coherent sheaves on a toric variety and the derived category of constructible sheaves on a torus. NCCC is discovered by Bondal [3] and formulated in terms of microlocal sheaf theory by Fang-Liu-Treumann-Zaslow [4] as follows.

Let $M$ be a free abelian group of finite rank and $N$ be its dual free abelian group. Let further $\Sigma$ be a smooth complete fan defined in $N_{\mathbb{R}}=N \otimes_{\mathbb{Z}} \mathbb{R}$ and $X_{\Sigma}$ be the toric variety defined by $\Sigma$. We write the bounded derived category of coherent sheaves on $X_{\Sigma}$ by $\mathrm{D}^{\mathrm{b}}\left(\operatorname{coh} X_{\Sigma}\right)$ and the bounded derived category of constructible sheaves on $M_{\mathbb{R}} / M$ by $\mathrm{D}_{c}^{\mathrm{b}}\left(M_{\mathbb{R}} / M\right)$. Here constructible sheaf means $\mathbb{R}$-constructible sheaf in the sense of $[6, \S 8.4]$. We define $\overline{\Lambda_{\Sigma}} \subset T^{*} M_{\mathbb{R}} / M$ as the coset of $\Lambda_{\Sigma}:=\bigcup_{\sigma \in \Sigma}\left(\sigma^{\perp}+M\right) \times(-\sigma) \subset M_{\mathbb{R}} \times N_{\mathbb{R}} \cong T^{*} M_{\mathbb{R}}$. We write the full subcategory of $\mathrm{D}_{c}^{\mathrm{b}}\left(M_{\mathbb{R}} / M\right)$ spanned by objects whose microsupports are contained in $\overline{\Lambda_{\Sigma}}$ by $\mathrm{D}_{c}^{\mathrm{b}}\left(M_{\mathbb{R}} / M, \overline{\Lambda_{\Sigma}}\right)$. It is known that there exists a fully-faithful functor

$$
\kappa_{\Sigma}: \mathrm{D}^{\mathrm{b}}\left(\operatorname{coh} X_{\Sigma}\right) \hookrightarrow \mathrm{D}_{c}^{\mathrm{b}}\left(M_{\mathbb{R}} / M, \overline{\Lambda_{\Sigma}}\right)
$$

which will be defined in (3.12).

Conjecture 1.1 (NCCC conjecture $[\mathbf{5}, \mathbf{1 1}]$ ). The functor $\kappa_{\Sigma}$ is an equivalence of triangulated categories

$$
\mathrm{D}^{\mathrm{b}}\left(\operatorname{coh} X_{\Sigma}\right) \cong \mathrm{D}_{c}^{\mathrm{b}}\left(M_{\mathbb{R}} / M, \overline{\Lambda_{\Sigma}}\right) .
$$

Received August 31, 2015. 
This conjecture is proved in special cases $([\mathbf{1 1}, \mathbf{1 0}]$, see also Theorem $3.2)$. The equivariant version of this conjecture is called the coherentconstructible correspondence and proved by Fang-Liu-TreumannZaslow [4].

In this paper, we prove Conjecture 1.1 in dimension 2 :

Theorem 1.2. Conjecture 1.1 holds for any 2-dimensional smooth complete fans.

Our proof is based on Theorem 1.3 below.

Theorem 1.3. Let $\Sigma$ be an $n$-dimensional smooth complete fan and $\hat{\Sigma}$ be its blow-up at a torus fixed point. Then there exists a semi-orthogonal decomposition

$$
\mathrm{D}_{c}^{\mathrm{b}}\left(M_{\mathbb{R}} / M, \overline{\Lambda_{\hat{\Sigma}}}\right) \cong\left\langle\mathbb{D}\left(p_{*} \mathbb{C}_{(n-1) \cdot Z}\right), \ldots, \mathbb{D}\left(p_{*} \mathbb{C}_{Z}\right), \mathrm{D}_{c}^{\mathrm{b}}\left(M_{\mathbb{R}} / M, \overline{\Lambda_{\Sigma}}\right)\right\rangle .
$$

Here $p: M_{\mathbb{R}} \rightarrow M_{\mathbb{R}} / M$ is the quotient map, $\mathbb{D}$ is the Verdier duality functor, and $Z$ is a locally closed subset of $M_{\mathbb{R}}$ which will be defined in (4.1). This formula is an analog of Orlov's theorem on the semiorthogonal decomposition of derived category of coherent sheaves under blowing-up $[\mathbf{9}, \mathbf{2}]$. In the situation of Theorem 1.3, Orlov's theorem says that the derived category of coherent sheaves on $X_{\hat{\Sigma}}$ has a semiorthogonal decomposition

$$
\mathrm{D}^{\mathrm{b}}\left(\operatorname{coh} X_{\hat{\Sigma}}\right) \cong\left\langle\mathcal{O}_{E}((n-1) E), \ldots, \mathcal{O}_{E}(E), \pi^{*} \mathrm{D}^{\mathrm{b}}\left(\operatorname{coh} X_{\Sigma}\right)\right\rangle
$$

where $\pi: X_{\hat{\Sigma}} \rightarrow X_{\Sigma}$ is the blow-up morphism and $E$ is the exceptional divisor. In Lemma 4.1 , we will prove $\kappa_{\hat{\Sigma}}\left(\mathcal{O}_{E}(k E)\right) \cong \mathbb{D}\left(p_{*} \mathbb{C}_{k Z}\right)[-n]$. Then one can identify Theorem 1.3 with Orlov's theorem via NCCC. Comparing the semi-orthogonal components, we have the following:

Theorem 1.4. Let $\Sigma$ be an $n$-dimensional smooth complete fan and $\hat{\Sigma}$ be its blow-up at a torus fixed point. Conjecture 1.1 holds for $\Sigma$ if and only if so is for $\hat{\Sigma}$.

In the case of toric surfaces, toric MMP and Theorem 1.4 allow us to reduce Conjecture 1.1 to the case of $\mathbb{P}^{1} \times \mathbb{P}^{1}$ which is already proved by Treumann [11].

Nadler and Zaslow $[\mathbf{8}, \mathbf{7}]$ identifies the derived Fukaya category of a cotangent bundle with the bounded derived category of constructible sheaves on its base space. By using this result, Fang-Liu-TreumannZaslow [5] relates NCCC with homological mirror symmetry. Combining their results with our results, we obtain a version of homological mirror symmetry for toric surfaces:

Corollary 1.5. Let $\Sigma$ be a 2-dimensional smooth complete fan. Then there exists an equivalence of triangulated categories

$$
\mathrm{D}^{\mathrm{b}}\left(\operatorname{coh} X_{\Sigma}\right) \cong \operatorname{DFuk}\left(T^{*} M_{\mathbb{R}} / M, \overline{\Lambda_{\Sigma}}\right) .
$$


The notations will be explained in Section 2.

This paper is organized as follows. In Section 2, we briefly recall microlocal sheaf theory of Kashiwara-Schapira [6]. In Section 3, we review an aspect of the NCCC and collect notations. In Section 4, we prove Theorem 1.3. Finally, in Section 5, we give a proof of Theorem 1.2 and 1.4.

Acknowledgments. I would like to express my gratitude to Professor Kazushi Ueda and Professor Shinobu Hosono for many comments and encouragements. I would like to thank Yuichi Ike and Fumihiko Sanda for having many stimulating discussions and giving many comments on this paper. I also thank Ike and Takahiro Saito for answering my questions on microlocal sheaf theory many times, Daisuke Inoue and Makoto Miura for many comments at the seminars. I also would like to thank for the referee for useful comments. This work was supported by the program for Leading Graduate Schools, MEXT, Japan.

\section{Backgrounds from microlocal sheaf theory}

Let $Y$ be a differentiable manifold and $\mathrm{D}^{\mathrm{b}}(\mathrm{Sh} Y)$ be the derived cat-

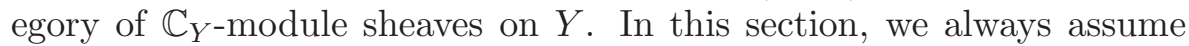
that $n \geq 2$.

Definition 2.1 ([6, Definition 5.1.2]). For $\mathcal{E} \in \mathrm{D}^{\mathrm{b}}(\mathrm{Sh} Y)$, the $m i$ crosupport $\mathrm{SS}(\mathcal{E})$ of $\mathcal{E}$ is a closed subset of $T^{*} Y$ defined as follows; for $(x, \xi) \in T^{*} Y,(x, \xi)$ is not contained in $\operatorname{SS}(\mathcal{E})$ if there exists an open neighborhood $V$ of $(x, \xi)$ such that for any $C^{1}$ function $\psi$ with $\operatorname{Graph}(d \psi) \subset V$ and

$$
\left(\mathbb{R} \Gamma_{\{y \mid \psi(y) \geq \psi(x)\}} \mathcal{E}\right)_{x} \simeq 0 .
$$

The microsupport detects the direction where the cohomology of the sheaf does not extend isomorphically.

For a cone $\gamma \subset N_{\mathbb{R}}$, we define the dual cone $\gamma^{\vee}$ as

$$
\gamma^{\vee}:=\left\{m \in M_{\mathbb{R}} \mid\langle m, n\rangle \geq 0 \text { for any } n \in \gamma\right\} \text {. }
$$

For a subset $Z \subset Y$, we write the interior of $Z$ by $\operatorname{Int}(Z)$ and the relative interior of $Z$ by $\operatorname{Relint}(Z)$. We say a closed convex cone $\gamma$ is proper (or strongly convex) if it satisfies $\gamma \cap(-\gamma)=\{0\}$.

Proposition $2.2\left(\left[6\right.\right.$, Proposition 5.1.1]). For $\mathcal{E} \in \mathrm{D}^{\mathrm{b}}\left(\mathrm{Sh} \mathbb{R}^{n}\right)$ and $\left(x_{0}, \xi_{0}\right) \in T^{*} \mathbb{R}^{n},\left(x_{0}, \xi_{0}\right) \in \mathrm{SS}(\mathcal{E})$ is equivalent to the following: For any neighborhood $V$ of $x_{0}$, any positive integer $\epsilon$, and any proper convex cone $\delta$ with $\xi_{0} \in \operatorname{Int}\left(\delta^{\vee}\right)$, there exists $x \in V$ such that

$$
\mathbb{R} \Gamma(H \cap(x-\delta), \mathcal{E}) \stackrel{\nsucceq}{\rightarrow} \mathbb{R} \Gamma(L \cap(x-\delta), \mathcal{E}),
$$


where

$$
\begin{aligned}
H & :=\left\{y \in \mathbb{R}^{n} \mid\left\langle y-x_{0}, \xi_{0}\right\rangle \geq-\epsilon\right\}, \\
L & :=\left\{y \in \mathbb{R}^{n} \mid\left\langle y-x_{0}, \xi_{0}\right\rangle=-\epsilon\right\} .
\end{aligned}
$$

Theorem 2.3 (the non-characteristic deformation lemma [6, Proposition 2.7.2]). Let $I$ be an open interval in $\mathbb{R},\left\{U_{s}\right\}_{s \in I}$ be a family of open subsets in $Y$, and $\mathcal{E} \in \mathrm{D}^{\mathrm{b}}(\mathrm{Sh} Y)$ satisfying the following:

(1) $U_{s}=\bigcup_{t<s} U_{t}$ for any $s \in I$.

(2) $U_{t} \backslash U_{s}$ is relatively compact for any $(s, t) \in I^{2}$.

(3) $\left(\mathbb{R} \Gamma_{Y \backslash U_{t}} \mathcal{E}\right)_{x} \simeq 0$ for any $s \leq t$ and $x \in \bigcap_{u>s} \operatorname{Cl}\left(U_{u} \backslash U_{s}\right) \backslash U_{t}$ where $\mathrm{Cl}$ denotes taking closure.

Then, we have $\mathbb{R} \Gamma\left(\bigcup_{t \in I} U_{t}, \mathcal{E}\right) \stackrel{\sim}{\rightarrow} \mathbb{R} \Gamma\left(U_{s}, \mathcal{E}\right)$ for any $s \in I$.

Theorem 2.3 holds even if $Y$ is not a manifold but $Y$ is simply a Hausdorff topological space.

For a subset $Z$ of $Y$, we define the strict normal cone $N_{x} Z \subset T_{x} Y$ of $Z$ at $x \in Y$ as follows; the tangent vector $\xi \in T_{x} Y$ is not contained in $N_{x} Z$ if there exists a local coordinate $U$ of $x$ and two sequences $\left\{x_{i}\right\}_{i \in \mathbb{N}} \subset U \backslash Z$ and $\left\{y_{i}\right\}_{i \in \mathbb{N}} \subset U \cap Z$ satisfying convergence conditions; $x_{i}, y_{i} \rightarrow x$ and $x_{i}-y_{i} /\left|x_{i}-y_{i}\right| \rightarrow \xi /|\xi|$ in $U$. We define the conormal cone $N_{x}^{*} Z$ of $Z$ at $x$ as the dual cone $N_{x} Z^{\vee}$ of $N_{x} Z$.

Lemma 2.4. Let $\gamma$ be a closed convex cone in $\mathbb{R}^{n}$. Then we have

1) $N_{0}^{*} \gamma=\gamma^{\vee}$, and

2) $N_{0}^{*}\left(\mathbb{R}^{n} \backslash \gamma\right)=-\gamma^{\vee}$.

Proof. These are clear from the definition of conormal cone. q.e.d.

We say a subset $Z$ of $\mathbb{R}^{n}$ is polyhedral if it is defined by finite linear inequalities.

Lemma 2.5. Let $Z, W \subset \mathbb{R}^{n}$ be a polyhedral subset. Then we have

$$
N_{x}^{*}(Z \cap W)=N_{x}^{*} Z+N_{x}^{*} W
$$

for $x \in Z \cap W$.

Proof. This is also clear from the definition of conormal cone. q.e.d.

Figure 1 shows some examples of Lemma 2.4.

Lemma 2.6 ([6, Corollary 5.4.9]). We use the same notation as in the previous lemma. For $\mathcal{E} \in \mathrm{D}^{\mathrm{b}}(\operatorname{Sh} Y)$, we assume that $\operatorname{SS}(\mathcal{E}) \cap N_{x}^{*} Z \subset$ $\{(x, 0)\}$. Then, we have $\left(\mathbb{R} \Gamma_{Z}(\mathcal{E})\right)_{x} \simeq 0$.

Lemma 2.7. Let $\gamma \subset \mathbb{R}^{n}$ be an $n$-dimensional closed polyhedral convex cone. We assume that there exists a proper $n$-dimensional closed polyhedral convex cone $\delta \subset \mathbb{R}^{n}$ such that $-\delta \cap \gamma=\{0\}$. For $\mathcal{E} \in$ $\mathrm{D}^{\mathrm{b}}\left(\mathrm{Sh} \mathbb{R}^{n}\right)$, we further assume that there exists a neighborhood $U$ of 0 

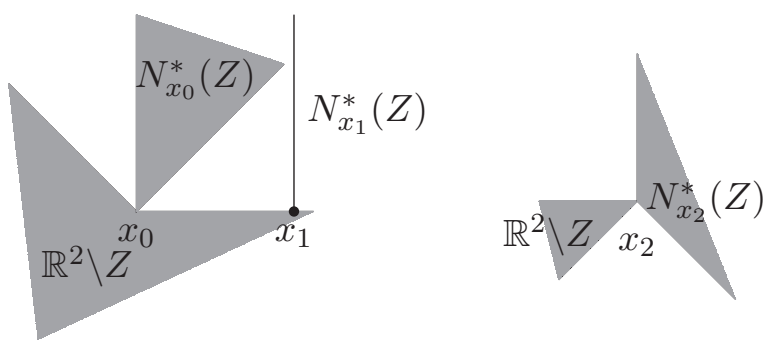

Figure 1. Some examples of conormal cone.

such that $\operatorname{SS}(\mathcal{E}) \cap U \times\left(\operatorname{Relint}\left(\gamma^{\vee}\right)+\delta^{\vee}\right)=\varnothing$ under the canonical identification $T^{*} \mathbb{R}^{n} \cong \mathbb{R}^{n} \times \mathbb{R}^{n}$. Then, we have $\left(\mathbb{R} \Gamma_{\gamma}(\mathcal{E})\right)_{0} \simeq 0$.

Proof. We take a family of closed convex polyhedral cones $\left\{\gamma_{s}\right\}_{s \in[0, \infty)}$ having the following properties;

(i) $\gamma \backslash\{0\} \subset \operatorname{Int}\left(\gamma_{s}\right)$ for any $s \in[0, \infty)$,

(ii) $\gamma_{s} \subset \gamma_{t}$ for $s \geq t$,

(iii) $\bigcap_{s \in[0, \infty)} \gamma_{s}=\gamma$,

(iv) $-\delta \cap \gamma_{t}=\{0\}$.

Take $x \in \operatorname{Int}(\delta)$, then $0 \in(-\operatorname{Int}(\delta)+c x) \cap \gamma_{t}$ for any $c \in(0,1)$ and $t \in[0, \infty)$. The assumption (iv) tells us that $\left\{(-\delta+c x) \cap \gamma_{t}\right\}_{c \in(0,1)}$ forms a neighborhood system of 0 in $\gamma_{t}$. Hence we can take sufficiently small $c$ such that $(-\delta+c x) \cap \gamma_{t} \subset U$. In the following, we rewrite $c x$ as $x$.

We define

$$
V_{s}:= \begin{cases}\varnothing & \text { for } s<0, \\ (\operatorname{Int}(-\delta)+s x) \cap U & \text { for } s \in[0,1) .\end{cases}
$$

Fix some $s \in[0,1]$ and $t \in[0, \infty)$ we define

$$
U_{u}:=V_{u s} \cup\left(V_{s} \backslash \gamma_{t}\right) \text {, }
$$

for $u \in(-1,1)$. Here $V_{u s}=\varnothing$ for $u s<0$. Then we obtain a family of open subsets $\mathcal{U}:=\left\{U_{u}\right\}_{u \in(-1,1)}$. Since $(t-s) \cdot x \in \operatorname{Int}(\delta)$ for $s<t \in[0,1)$, we have $V_{s} \subset V_{t}$. Hence we have (1) of Theorem 2.3 for $\mathcal{U}$.

We also have $(2)$ of Theorem 2.3 since $(-\delta+x) \cap \gamma_{t} \subset U$.

For any $a \leq b$, the set $\bigcap_{c>a} \mathrm{Cl}\left(U_{c} \backslash U_{a}\right) \backslash U_{b}$ is empty or $\partial V_{u s} \cap \gamma_{t}$. At $x \in \partial V_{u s} \cap \gamma_{t}$, we have

$$
\begin{aligned}
N_{x}^{*}\left(\mathbb{R}^{n} \backslash U_{u}\right) & =N_{x}^{*}\left(\left(\mathbb{R}^{n} \backslash V_{u s}\right) \cap\left(\mathbb{R}^{n} \backslash\left(V_{s} \backslash \gamma_{t}\right)\right)\right) \\
& =N_{x}^{*}\left(\mathbb{R}^{n} \backslash V_{u s}\right)+N_{x}^{*}\left(\mathbb{R}^{n} \backslash\left(V_{s} \backslash \gamma_{t}\right)\right) \\
& =-N_{x}^{*}\left(V_{u s}\right)+N_{x}^{*}\left(\gamma_{t}\right) \\
& \subset \delta^{\vee}+\gamma_{t}^{\vee},
\end{aligned}
$$

by Lemma 2.4 and Lemma 2.5. By the assumption (i), $\gamma_{t}^{\vee} \backslash\{0\}$ is contained in $\operatorname{Relint}\left(\gamma^{\vee}\right)$ for $t \in[0, \infty)$. Hence, we have $U \times \gamma_{t}^{\vee} \cap \operatorname{SS}(\mathcal{E}) \subset\{0\}$. 
Since $\partial V_{s} \cap \gamma_{t} \subset U, \operatorname{SS}(\mathcal{E}) \cap U \times\left(\delta^{\vee}+\gamma_{t}^{\vee}\right)=\{0\}$. Then, by Lemma 2.6, $\left\{U_{u}\right\}_{u \in(-1,-1)}$ satisfy (3) of Theorem 2.3. Hence we can use Theorem 2.3 and we have

$$
\mathbb{R} \Gamma\left(V_{s}, \mathcal{E}\right) \stackrel{\simeq}{\rightrightarrows} \mathbb{R} \Gamma\left(V_{s} \backslash \gamma_{t}, \mathcal{E}\right) .
$$

In other words, we have $\mathbb{R} \Gamma_{\gamma_{t}}\left(V_{s}, \mathcal{E}\right) \simeq 0$ for any $s$ and $t$.

Taking the limit with respect to $t$, we have $\mathbb{R} \Gamma_{\gamma}\left(V_{s}, \mathcal{E}\right) \simeq 0$ for any $s \in[0,1)$. Since $-\delta \cap \gamma=\{0\}$, we know that $\left\{V_{s} \cap \gamma\right\}_{s \in[0,1)}$ is an open neighborhood system of 0 in $\gamma$. Finally, we have $\left(\mathbb{R} \Gamma_{\gamma}(\mathcal{E})\right)_{0} \simeq 0$. q.e.d.

Let $E \cong \mathbb{R}^{n}$ be a vector space and $\gamma \subset E$ be an $n$-dimensional closed polyhedral convex cone. For a face $\tau$ of $\gamma^{\vee}$, we define a subset $R_{\tau}$ of $E$ by

$$
R_{\tau}:=\bigcap_{\substack{\tau \prec \sigma \prec \gamma^{\vee} \\ \tau \neq \sigma}}\left(\tau^{\perp} \backslash \sigma^{\perp}\right),
$$

where $\tau \prec \sigma$ means $\tau$ is a face of $\sigma$.

Lemma 2.8. Let $\gamma \subset E \cong \mathbb{R}^{n}$ be an n-dimensional closed polyhedral convex cone. Let further $\delta$ be an n-dimensional proper polyhedral closed convex cone. For $\mathcal{E} \in \mathrm{D}^{\mathrm{b}}(\mathrm{Sh} E)$, we assume the following:

(i) There exists a neighborhood $V$ of 0 such that $(V \backslash \gamma) \times\left(\delta^{\vee}+\gamma^{\vee}\right) \cap$ $\operatorname{SS}(\mathcal{E}) \subset(V \backslash \gamma) \times\{0\} \cup \bigsqcup_{0 \neq \tau \prec \gamma \vee} R_{\tau} \times \tau$.

(ii) There exists $\xi_{0} \in \operatorname{Relint}\left(\gamma^{\vee}\right) \cap \operatorname{Int}\left(\delta^{\vee}\right)$ such that $\left(0, \xi_{0}\right) \in \operatorname{SS}(\mathcal{E})$. In particular, $\gamma \cap(-\delta)=\{0\}$.

(iii) $\left(\gamma^{\vee} \backslash \operatorname{Relint}\left(\gamma^{\vee}\right)\right) \cap \delta^{\vee}=\{0\}$.

(iv) $\operatorname{Int}(\gamma) \cap \operatorname{Int}(\delta) \neq \varnothing$.

Then, for any neighborhood $U$ of 0 , there exists $x \in U$ such that $\mathbb{R} \Gamma_{\gamma}(-\delta+x, \mathcal{E}) \not 0$.

Proof. We note that (ii) and (iv) imply a family of closed subsets $\{(x-\delta) \cap \gamma \mid x \in \operatorname{Int}(\gamma) \cap \operatorname{Int}(\delta)\}$ forms a neighborhood system of 0 in $\gamma$. Combining with $\xi_{0} \in \operatorname{Int}\left(\delta^{\vee}\right)$ from (ii), we can take a sufficiently small $\epsilon$ and $x_{0} \in \operatorname{Int}(\gamma) \cap \operatorname{Int}(\delta)$ such that $\bar{W}:=\left(x_{0}-\delta\right) \cap H \subset V \cap U$ where

$$
H:=\left\{y \in V \mid\left\langle y, \xi_{0}\right\rangle \geq-\epsilon\right\} .
$$

We define an open neighborhood $W$ of 0 by $W:=\operatorname{Int}(\bar{W})$. Proposition 2.2 and the property $\left(0, \xi_{0}\right) \in \mathrm{SS}(\mathcal{E})$ from (ii) ensures that there exists $x \in W$ such that

$$
\mathbb{R} \Gamma(H \cap(x-\delta), \mathcal{E}) \stackrel{\nsucceq}{\rightarrow} \mathbb{R} \Gamma(L \cap(x-\delta), \mathcal{E}),
$$

where

$$
L:=\left\{y \in V \mid\left\langle y, \xi_{0}\right\rangle=-\epsilon\right\} .
$$

By the definition of $W$, it follows that $H \cap(x-\delta), L \cap(x-\delta) \subset V$. 
Let us assume that $(x-\delta) \cap \gamma$ were empty. Then take $y_{0} \in \operatorname{Relint}((x-$ $\delta \cap \gamma) \cap L)$. From $\xi_{0} \in \operatorname{Relint}\left(\gamma^{\vee}\right)$ of (ii), we can define a family of open subsets $\mathcal{V}:=\left\{V_{s}\right\}_{s \in(-1,1+\alpha)}$ of $H$ with

$$
V_{s}:=\left(s x+(1-s) y_{0}-\operatorname{Int}(\delta)\right) \cap H \text { when } s \in(0,1+\alpha),
$$

where $\alpha>0$ is taken to be sufficiently small to satisfy $V_{1+\alpha} \subset V \backslash \gamma$. The family $\mathcal{V}$ clearly satisfies (1) and (2) of Theorem 2.3. For $s \geq t$, the subset $\bigcap_{u>s} \mathrm{Cl}\left(V_{u} \backslash V_{s}\right) \backslash V_{t}$ is $\varnothing$ or $\left(s x+(1-s) y_{0}-\partial \delta\right) \cap H$. Hence the normal cone $N_{y}^{*}\left(V_{1+\alpha} \backslash V_{s}\right)$ is contained in $\xi_{0}+\delta^{\vee}=\delta^{\vee}$ for $y \in$ $\bigcap_{u>s} \mathrm{Cl}\left(V_{u} \backslash V_{s}\right) \backslash V_{t}$. Since $V_{1+\alpha} \subset V \backslash \gamma$, (i) and (iii) tell us that $\operatorname{SS}(\mathcal{E}) \cap$ $N_{y}^{*}\left(V_{1+\alpha} \backslash V_{s}\right)=\{y\} \times\{0\}$ for $y \in \bigcap_{u>s} \mathrm{Cl}\left(V_{u} \backslash V_{s}\right) \backslash V_{t}$. By Lemma 2.6, $\mathcal{V}$ satisfies (3) of Theorem 2.3. Theorem 2.3 says that

$$
\mathbb{R} \Gamma\left(V_{1+\alpha}, \mathcal{E}\right) \stackrel{\simeq}{\longrightarrow} \mathbb{R} \Gamma\left(L \cap\left((1+\alpha) x-\alpha y_{0}-\delta\right), \mathcal{E}\right),
$$

for any sufficiently small $\alpha$. Taking $\alpha \rightarrow 0$, we have

$$
\mathbb{R} \Gamma(H \cap(x-\delta), \mathcal{E}) \stackrel{\simeq}{\rightarrow} \mathbb{R} \Gamma(L \cap(x-\delta), \mathcal{E}) .
$$

This contradicts to $(2.13)$, hence we have $(x-\delta) \cap \gamma \neq \varnothing$.

We again define a family of open subsets $\mathcal{V}_{\alpha}^{\prime}:=\left\{V_{s, \alpha}^{\prime}\right\}_{s \in(-1,1)}$ of $H$ with

$$
\begin{aligned}
V_{s, \alpha}^{\prime}:= & \left((s-1)(1+\alpha) x+(1-s) y_{0}\right. \\
& +(((1+\alpha) x-\operatorname{Int}(\delta) \backslash \gamma)) \cap H \text { when } s \in(0,1),
\end{aligned}
$$

where $\alpha$ is taken to be sufficiently small to satisfy $V_{1, \alpha}^{\prime} \subset V$. It is clear that the family $\mathcal{V}_{\alpha}^{\prime}$ satisfies (1) and (2) of Theorem 2.3. For $s$, we define two sets:

$$
Z_{1}:=\left((s-1)(1+\alpha) x+(1-s) y_{0}+(((1+\alpha) x-\partial \delta) \backslash \gamma)\right) \cap H
$$

$$
Z_{2}:=(s-1)(1+\alpha) x+(1-s) y_{0}+(((1+\alpha) x-\delta) \cap \partial \gamma) .
$$

Then, for $s \leq t, \bigcap_{u>s} \mathrm{Cl}\left(V_{u, \alpha}^{\prime} \backslash V_{s, \alpha}^{\prime}\right) \backslash V_{t, \alpha}^{\prime}$ is $\varnothing$ or $Z_{1} \sqcup Z_{2}$. If $y \in$ $Z_{1}$, the normal cone $N_{y}^{*}\left(V_{1, \alpha}^{\prime} \backslash V_{s, \alpha}^{\prime}\right)$ is contained in $\delta^{\vee}$, hence we have $\mathrm{SS}(\mathcal{E}) \cap N_{y}^{*}\left(V_{1, \alpha}^{\prime} \backslash V_{s, \alpha}^{\prime}\right)=\{y\} \times\{0\}$ by (i) and (iii). On the other hand, we have a decomposition $Z_{2}=\bigsqcup_{\tau \supsetneqq} \vee W_{\tau}$ where $W_{\tau}$ is defined by

$$
W_{\tau}:=(s-1)(1+\alpha) x+(1-s) y_{0}+\left(((1+\alpha) x-\delta) \cap R_{\tau} \cap \gamma\right) .
$$

Since $y_{0}-x \in-\operatorname{Int}(\gamma)$, we have $\left\langle y_{0}-x, \gamma^{\vee} \backslash\{0\}\right\rangle<0$. Then we have $W_{\tau} \cap \bigcup_{\tau \prec \sigma \prec \gamma^{\vee}} R_{\sigma} \cup \bigcup_{\sigma \prec \tau} R_{\sigma}=\varnothing$. Moreover, if $\sigma$ is not a face of $\tau$ and $\tau$ is not a face of $\sigma, \sigma \cap \tau$ is a proper face of $\sigma$ and $\tau$. For $y \in W_{\tau}$, we have

$$
N_{y}^{*}\left(V_{1, \alpha}^{\prime} \backslash V_{s, \alpha}^{\prime}\right)=\{y\} \times\left(f\left(\delta^{\vee}\right)+\tau\right)
$$


where $f\left(\delta^{\vee}\right)$ is one of the faces of $\delta^{\vee}$. By (iii), we can see $\sigma \cap\left(\delta^{\vee}+\tau\right)$ is a proper face of $\sigma$. Taking intersection of $N_{y}^{*}\left(V_{1, \alpha}^{\prime} \backslash V_{s, \alpha}^{\prime}\right)$ with the inclusion relation of (i), we have

$$
\operatorname{SS}(\mathcal{E}) \cap \operatorname{Relint}\left(N_{y}^{*}\left(V_{1, \alpha}^{\prime} \backslash V_{s, \alpha}^{\prime}\right)\right)=\varnothing .
$$

By Lemma 2.7, the family $\mathcal{V}_{\alpha}^{\prime}$ satisfies (3) of Theorem 2.3 and we have

$$
\mathbb{R} \Gamma\left(H \cap V_{1, \alpha}^{\prime}, \mathcal{E}\right) \stackrel{\simeq}{\rightarrow} \mathbb{R} \Gamma\left(L \cap\left((1+\alpha) x-\alpha y_{0}-\delta\right), \mathcal{E}\right) .
$$

Take $\alpha \rightarrow 0$, then (2.24) becomes

$$
\mathbb{R} \Gamma(H \cap(x-\delta) \backslash \gamma, \mathcal{E}) \stackrel{\simeq}{\rightarrow} \mathbb{R} \Gamma(L \cap(x-\delta), \mathcal{E}) .
$$

Combining this with (2.13), we have

$$
\mathbb{R} \Gamma(H \cap(x-\delta), \mathcal{E}) \stackrel{f}{\rightarrow} \mathbb{R} \Gamma(H \cap(x-\delta) \backslash \gamma, \mathcal{E}) .
$$

Hence we have $\mathbb{R} \Gamma_{\gamma}(H \cap(x-\delta), \mathcal{E}) \not 00$. Since $\gamma \subset H$, we conclude $\mathbb{R} \Gamma_{\gamma}(x-\delta, \mathcal{E}) \not 0$. q.e.d.

\section{A review of NCCC}

Let $M$ be a free abelian group of rank $n$ and $N$ be the dual free abelian group of $M$. We consider a smooth complete fan $\Sigma$ defined in $N_{\mathbb{R}}:=N \otimes_{\mathbb{Z}} \mathbb{R}$ and the associated toric variety $X_{\Sigma}$. For $\sigma \in \Sigma$, we write the corresponding affine toric subvariety of $X_{\Sigma}$ by $U_{\sigma}$ and the open immersion by $i_{\sigma}: U_{\sigma} \hookrightarrow X_{\Sigma}$. The theta quasi-coherent sheaf associated to $\sigma \in \Sigma$ is

$$
\overline{\Theta^{\prime}}(\sigma):=\mathcal{O}_{\sigma}:=i_{\sigma *} \mathcal{O}_{U_{\sigma}} \in \mathrm{D}^{\mathrm{b}}\left(\mathrm{Q} \operatorname{coh} X_{\Sigma}\right),
$$

where $\mathrm{D}^{\mathrm{b}}\left(\mathrm{Q} \operatorname{coh} X_{\Sigma}\right)$ is the bounded derived category of quasi-coherent sheaves. It is known that $\mathrm{D}^{\mathrm{b}}\left(\operatorname{coh} X_{\Sigma}\right) \subset\left\langle\overline{\Theta^{\prime}}(\sigma)\right\rangle_{\sigma \in \Sigma}$, where $\langle\cdot\rangle$ denotes the generated full subcategory [11, Proposition 2.6].

We define the theta quasi-constructible sheaf associated to $\sigma \in \Sigma$ as

$$
\bar{\Theta}(\sigma):=p ! \mathbb{D}\left(\mathbb{C}_{\sigma^{\vee}}\right) \in \mathrm{D}_{q c}^{\mathrm{b}}\left(M_{\mathbb{R}} / M\right),
$$

where $p: M_{\mathbb{R}} \rightarrow M_{\mathbb{R}} / M$ is the quotient map, $\mathbb{D}: \mathrm{D}_{q c}^{\mathrm{b}}\left(M_{\mathbb{R}}\right) \rightarrow \mathrm{D}_{q c}^{\mathrm{b}}\left(M_{\mathbb{R}}\right)^{o p}$ is the Verdier duality functor, $\mathbb{C}_{\sigma \vee}$ is the zero-extension of the constant sheaf on $\sigma^{\vee}$, and $\mathrm{D}_{q c}^{\mathrm{b}}\left(M_{\mathbb{R}} / M\right)$ is the bounded derived category of quasiconstructible sheaves of $\mathbb{C}$-modules. Here, quasi-constructible (weakly constructible in $[\mathbf{6}]$ ) means that it is locally constant along some stratification but not necessarily of finite rank.

For $m \in \tau^{\vee}$, one can define $\theta_{m}^{\prime} \in \operatorname{Hom}_{\mathrm{D}^{\mathrm{b}}\left(\mathrm{Qcoh} X_{\Sigma}\right)}^{0}\left(\overline{\Theta^{\prime}}(\sigma), \overline{\Theta^{\prime}}(\tau)\right)$ the multiplication by the character $\chi^{m}$;

$$
\theta_{m}^{\prime}: \overline{\Theta^{\prime}}(\sigma) \stackrel{\times \chi^{m}}{\longrightarrow} \overline{\Theta^{\prime}}(\tau) .
$$


This correspondence induces an isomorphism

$\operatorname{Hom}_{\mathrm{D}^{\mathrm{b}}\left(\mathrm{Qcoh}_{\Sigma}\right)}^{i}\left(\overline{\Theta^{\prime}}(\sigma), \overline{\Theta^{\prime}}(\tau)\right) \cong \begin{cases}\mathbb{C}\left[\tau^{\vee} \cap M\right] & \text { when } \sigma \supset \tau \text { and } i=0, \\ 0 & \text { otherwise. }\end{cases}$

Similarly, for $m \in \tau^{\vee}$, one can define $\theta_{m} \in \operatorname{Hom}_{\mathrm{D}_{c}^{\mathrm{b}}\left(M_{\mathbb{R}} / M\right)}^{0}(\bar{\Theta}(\sigma), \bar{\Theta}(\tau))$ as the composition

$$
\theta_{m}: \bar{\Theta}(\sigma)=p_{!} \mathbb{D}\left(\mathbb{C}_{\sigma^{\vee}}\right)=p_{!} \mathbb{D}\left(\mathbb{C}_{\sigma^{\vee}+m}\right) \stackrel{p_{!} \mathbb{D}\left(r_{\sigma^{\vee}+m}^{\tau^{\vee}}\right)}{\longrightarrow} \bar{\Theta}(\tau),
$$

where $\chi^{m}$ is the character corresponding to $m$ and $r_{\sigma^{\vee}+m}^{\tau^{\vee}}: \mathbb{C}_{\tau^{\vee}} \rightarrow \mathbb{C}_{\sigma^{\vee}+m}$ is the restriction map. This correspondence induces an isomorphism

$$
\operatorname{Hom}_{\mathrm{D}_{c}^{\mathrm{b}}\left(M_{\mathbb{R}} / M\right)}^{i}(\bar{\Theta}(\sigma), \bar{\Theta}(\tau)) \cong \begin{cases}\mathbb{C}\left[\tau^{\vee} \cap M\right] & \text { when } \sigma \supset \tau \text { and } i=0, \\ 0 & \text { otherwise. }\end{cases}
$$

The category $\Gamma\left(\overline{\Lambda_{\Sigma}}\right)$ is a dg-category whose set of objects is $\Sigma$ and Hom-spaces are defined by

$$
\operatorname{hom}_{\Gamma\left(\overline{\Lambda_{\Sigma}}\right)}^{i}(\sigma, \tau):= \begin{cases}\mathbb{C}\left[\tau^{\vee} \cap M\right] & \text { when } \sigma \supset \tau \text { and } i=0, \\ 0 & \text { otherwise, }\end{cases}
$$

with trivial differentials. We write the full sub dg-category of $\mathrm{D}^{d g}\left(\mathrm{Q} \operatorname{coh} X_{\Sigma}\right)\left(\operatorname{resp} . \mathrm{D}_{q c}^{d g}\left(M_{\mathbb{R}} / M, \overline{\Lambda_{\Sigma}}\right)\right)$ spanned by $\left\{\overline{\Theta^{\prime}}(\sigma)\right\}_{\sigma \in \Sigma}$ (resp. $\left.\{\bar{\Theta}(\sigma)\}_{\sigma \in \Sigma}\right)$ by $\bar{\Theta}_{d g}^{\prime}$ (resp. $\bar{\Theta}_{d g}$ ). Then we have two quasi-equivalences of dg-categories

$$
\begin{aligned}
\Gamma\left(\overline{\Lambda_{\Sigma}}\right) & \rightarrow \overline{\Theta^{\prime}} d g, \\
\Gamma\left(\overline{\Lambda_{\Sigma}}\right) & \rightarrow \bar{\Theta}_{d g} .
\end{aligned}
$$

Hence, we also have the quasi-equivalence of perfect $\mathrm{dg}$-modules of $\mathrm{dg}$ categories $\operatorname{Per}_{d g} \bar{\Theta}_{d g} \simeq \operatorname{Per}_{d g} \bar{\Theta}_{d g}$. We write the equivalence induced on the homotopy categories by

$$
K_{\Sigma}:\left\langle\overline{\Theta^{\prime}}\right\rangle_{\sigma \in \Sigma} \cong H^{0}\left(\operatorname{Per}_{d g}{\overline{\Theta^{\prime}}}_{d g}\right) \cong H^{0}\left(\operatorname{Per}_{d g} \bar{\Theta}_{d g}\right) \cong\langle\bar{\Theta}\rangle_{\sigma \in \Sigma}
$$

By the definition, $K_{\Sigma}$ sends $\overline{\Theta^{\prime}}(\sigma)$ to $\bar{\Theta}(\sigma)$ and $\theta_{m}^{\prime}$ to $\theta_{m}[\mathbf{1 1}$, Theorem $2.3]$.

To describe NCCC, we identify $M_{\mathbb{R}} \times N_{\mathbb{R}} \cong T^{*} M_{\mathbb{R}}$ and define

$$
\Lambda_{\Sigma}:=\bigcup_{\sigma \in \Sigma}\left(\sigma^{\perp}+M\right) \times(-\sigma) \subset T^{*} M_{\mathbb{R}}
$$

and $\overline{\Lambda_{\Sigma}} \subset M_{\mathbb{R}} / M \times N_{\mathbb{R}} \cong T^{*} M_{\mathbb{R}} / M$ as the image of the $\Lambda_{\Sigma}$ under the projection $\tilde{p}: T^{*} M_{\mathbb{R}} \rightarrow T^{*} M_{\mathbb{R}} / M$. We write the full subcategory of $\mathrm{D}_{c}^{\mathrm{b}}\left(M_{\mathbb{R}} / M\right)$ whose objects have microsupports in $\overline{\Lambda_{\Sigma}}$ by $\mathrm{D}_{c}^{\mathrm{b}}\left(X_{\Sigma}, \overline{\Lambda_{\Sigma}}\right)$. Treumann showed that the essential image of $\mathrm{D}^{\mathrm{b}}\left(\operatorname{coh} X_{\Sigma}\right)$ by $K_{\Sigma}$ is 
contained in $\mathrm{D}_{c}^{\mathrm{b}}\left(M_{\mathbb{R}} / M, \overline{\Lambda_{\Sigma}}\right)[\mathbf{1 1}$, Proposition 2.7]. Hence we obtain the following functor

$$
\kappa_{\Sigma}:=\left.K_{\Sigma}\right|_{\mathrm{D}^{\mathrm{b}}\left(\operatorname{coh} X_{\Sigma}\right)}: \mathrm{D}^{\mathrm{b}}\left(\operatorname{coh} X_{\Sigma}\right) \rightarrow \mathrm{D}_{c}^{\mathrm{b}}\left(M_{\mathbb{R}} / M, \overline{\Lambda_{\Sigma}}\right) .
$$

The functor $\kappa_{\Sigma}$ is fully-faithful, since it is a restriction of an equivalence.

Conjecture 1.1 is motivated by homological mirror symmetry. By the result of Nadler-Zaslow [8] and Nadler [7], the derived Fukaya category $\operatorname{DFuk}\left(M_{\mathbb{R}} / M\right)$ of $M_{\mathbb{R}} / M$ and the bounded derived category of constructible sheaves $\mathrm{D}_{c}^{\mathrm{b}}\left(M_{\mathbb{R}} / M\right)$ on $M_{\mathbb{R}} / M$ are equivalent:

Theorem 3.1 (Nadler-Zaslow [8], Nadler [7]). For a real analytic manifold $X$, there exists an equivalence of triangulated categories

$$
\mathrm{D}_{c}^{\mathrm{b}}(X) \cong \operatorname{DFuk}\left(T^{*} X\right) \text {. }
$$

Fang-Liu-Treumann-Zaslow [5] defined DFuk $\left(T^{*} M_{\mathbb{R}} / M, \overline{\Lambda_{\Sigma}}\right)$ the essential image of $\mathrm{D}_{c}^{\mathrm{b}}\left(M_{\mathbb{R}} / M, \overline{\Lambda_{\Sigma}}\right)$ under Nadler-Zaslow's equivalence. Combining Theorem 3.1 with Conjecture 1.1, we have a version of homological mirror symmetry for toric varieties.

There are some results on Conjecture 1.1. We say a smooth complete fan $\Sigma$ is a zonotopal unimodular fan if $\Sigma$ is obtained from a hyperplane arrangement and any linearly independent subset of the set of ray generators of $\Sigma$ can be extended to $\mathbb{Z}$-basis of $N$. We say a smooth complete fan $\Sigma$ is cragged when the following two conditions are satisfied:

1) For any subset $S$ of $\Sigma$, the cone hull of $S$ is a union of a subset of $\Sigma$.

2) For any linearly independent subset $B$ of the set of ray generators $R$, the lattice generated by $\operatorname{Cone}(B) \cap R$ has $B$ as a $\mathbb{Z}$-basis.

Theorem 3.2 (Scherotzke-Sibilla [10], Treumann [11]). Let $\Sigma$ be a smooth complete fan.

1) Conjecture 1.1 holds when $\Sigma$ is zonotopal unimodular [11, Corollary 4.5].

2) Conjecture 1.1 holds when $\Sigma$ is cragged [10, Theorem 6.11].

Both $\mathrm{D}^{\mathrm{b}}\left(\operatorname{coh} X_{\Sigma}\right)$ and $\mathrm{D}_{c}^{\mathrm{b}}\left(M_{\mathbb{R}} / M, \overline{\Lambda_{\Sigma}}\right)$ carry monoidal structures as follows;

1) $\otimes:=\otimes^{\mathbb{L}}$; derived tensor product in $\mathrm{D}^{\mathrm{b}}\left(\operatorname{coh} X_{\Sigma}\right)$,

2) $\star:=m_{!} \circ \nabla^{\mathbb{L}}$; the composition of

$$
\begin{aligned}
\mathrm{D}_{c}^{\mathrm{b}}\left(M_{\mathbb{R}} / M, \overline{\Lambda_{\Sigma}}\right) \times \mathrm{D}_{c}^{\mathrm{b}}\left(M_{\mathbb{R}} / M, \overline{\Lambda_{\Sigma}}\right) & \\
& \stackrel{\nabla^{\mathbb{L}}}{\rightarrow} \mathrm{D}_{c}^{\mathrm{b}}\left(M_{\mathbb{R}} / M \times M_{\mathbb{R}} / M, \overline{\Lambda_{\Sigma}} \times \overline{\Lambda_{\Sigma}}\right) \\
& \stackrel{m_{!}}{\longrightarrow} \mathrm{D}_{c}^{\mathrm{b}}\left(M_{\mathbb{R}} / M, \overline{\Lambda_{\Sigma}}\right),
\end{aligned}
$$

where $\nabla^{\mathbb{L}}$ is the exterior tensor product and $m$ is the multiplication map with respect to the group structure of $M_{\mathbb{R}} / M$. 
Some general properties of $\kappa_{\Sigma}$ are known.

Theorem 3.3 (Fang-Liu-Treumann-Zaslow [4], Treumann [11]). Let $\Sigma$ be a smooth complete fan and $\hat{\Sigma}$ be a smooth complete subdivision of $\Sigma$. Let further $\pi^{*}: \mathrm{D}^{\mathrm{b}}\left(\operatorname{coh} X_{\Sigma}\right) \rightarrow \mathrm{D}^{\mathrm{b}}\left(\operatorname{coh} X_{\hat{\Sigma}}\right)$ be the pull-back along $\pi: X_{\hat{\Sigma}} \rightarrow X_{\Sigma}$ which is the morphism associated to the subdivision. Then we have the following:

(1) There exists a natural equivalence $\kappa_{\hat{\Sigma}} \circ \pi^{*} \cong \iota \circ \kappa_{\Sigma}$ where $\iota$ is the inclusion functor $\mathrm{D}_{c}^{\mathrm{b}}\left(M_{\mathbb{R}} / M, \overline{\Lambda_{\Sigma}}\right) \rightarrow \mathrm{D}_{c}^{\mathrm{b}}\left(M_{\mathbb{R}} / M, \overline{\Lambda_{\hat{\Sigma}}}\right)$ implied by $\overline{\Lambda_{\Sigma}} \subset \overline{\Lambda_{\hat{\Sigma}}}$.

(2) The functor $\kappa_{\Sigma}$ is monoidal with respect to the monoidal structures $\otimes$ and $\star$.

(3) There exists a natural equivalence, $\mathbb{D} \circ \kappa_{\Sigma} \cong \alpha^{*} \circ \kappa_{\Sigma} \circ D$ where $D:=\mathbb{R} \mathcal{H o m}\left(-, \mathcal{O}_{X_{\Sigma}}\right)$ and $\alpha: M_{\mathbb{R}} / M \rightarrow M_{\mathbb{R}} / M$ is the inversion map $x \mapsto-x$.

\section{NCCC and blow-up formula}

Let $M$ be a free abelian group with $n:=\operatorname{rank} M \geq 2, N$ be the dual of $M$, and $\Sigma$ be a smooth complete fan defined in $N_{\mathbb{R}}$. We write a toric blow-up of $X_{\Sigma}$ centered at a torus-fixed point by $\pi: X_{\hat{\Sigma}} \rightarrow X_{\Sigma}$, the exceptional divisor by $j: E \rightarrow X_{\Sigma}$, and the corresponding ray by $\rho_{E} \in \hat{\Sigma}$. We write the unique cone which corresponds to the affine toric subvariety containing the blow-up point by $\sigma_{c} \in \Sigma$. The cone $\sigma_{c}$ is $n$ dimensional and we write edges of $\sigma_{c}$ by $\rho_{1}, \ldots, \rho_{n}$ and the ray generator of $\rho_{i}$ by $e_{i}$. Then, we have $e_{E}:=\sum_{i=1}^{n} e_{i}$ for the ray generator of $\rho_{E}$. Since $\sigma$ is smooth, $\left\{e_{i}\right\}_{i=1}^{n}$ forms a basis of $N$. The dual basis of $M$ is denoted by $\left\{e_{i}^{\vee}\right\}_{i=1}^{n}$.

We define a locally closed subset $Z \subset M_{\mathbb{R}}$ by

$$
Z:=\left\{m \in M_{\mathbb{R}} \mid\left\langle m, e_{E}\right\rangle \geq-1\right\} \cap\left\{m \in M_{\mathbb{R}} \mid\left\langle m, e_{i}\right\rangle<0 \text { for any } i\right\} .
$$

Also recall that the functor

$$
\kappa_{\hat{\Sigma}}: \mathrm{D}^{\mathrm{b}}\left(\operatorname{coh} X_{\Sigma}\right) \rightarrow \mathrm{D}_{c}^{\mathrm{b}}\left(M_{\mathbb{R}} / M, \overline{\Lambda_{\hat{\Sigma}}}\right) .
$$

To prove Theorem 1.3, we prepare the following lemma.

Lemma 4.1. For $k \geq 1$,

$$
\kappa_{\hat{\Sigma}}\left(\mathcal{O}_{E}(k E)\right) \simeq \mathbb{D}\left(p_{*} \mathbb{C}_{Z_{k}}\right)[-n],
$$

where $Z_{k}:=k \cdot Z$ and $p: M_{\mathbb{R}} \rightarrow M_{\mathbb{R}} / M$ is the quotient map.

Some examples of the subsets $Z_{k}$ are depicted in Figure 2.

Proof. To calculate $\kappa_{\hat{\Sigma}}\left(\mathcal{O}_{E}(k E)\right)$, we first consider the following resolution;

$$
0 \rightarrow \mathcal{O} \rightarrow \mathcal{O}(E) \rightarrow \mathcal{O}_{E}(E) \rightarrow 0
$$



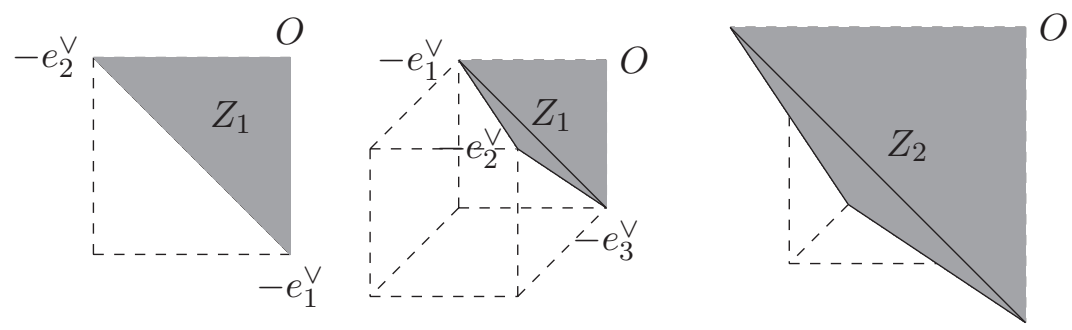

Figure 2. $Z_{1}$ in $n=2, Z_{1}$ and $Z_{2}$ in $n=3$.

where we write $\mathcal{O}_{X_{\hat{\Sigma}}}$ by $\mathcal{O}$.

Next, we take Čech resolutions:

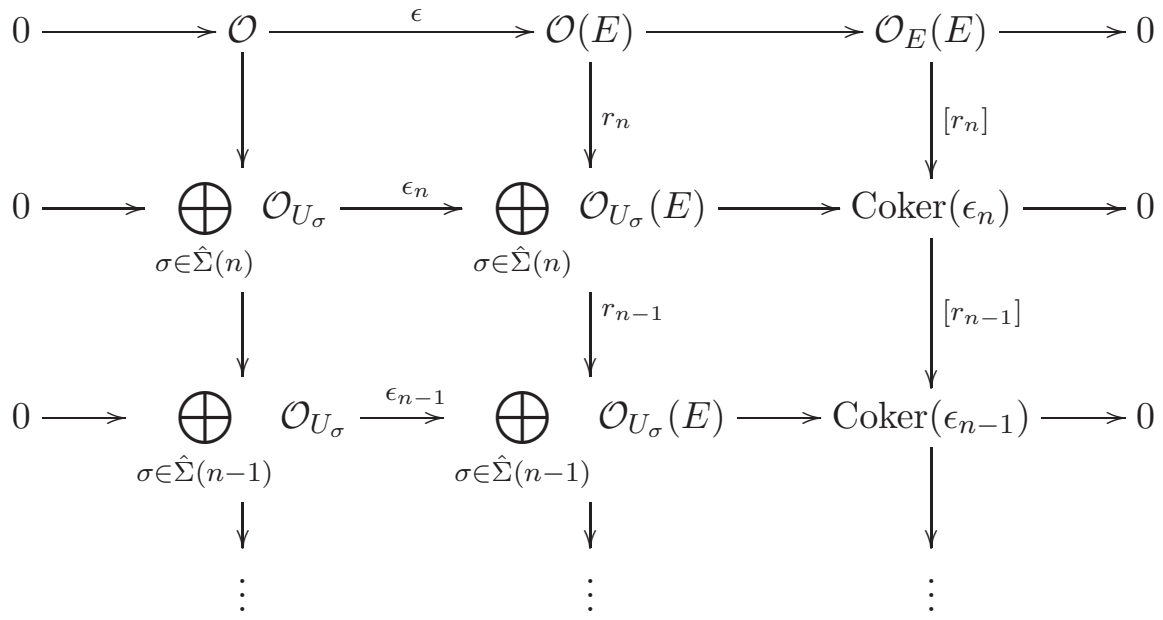

Hereafter, we will ignore cones in $\hat{\Sigma}$ which do not contain $\rho_{E}$, since they are irrelevant for the calculation of $\mathcal{O}_{E}(E)$ in the above diagram.

By definition, $\mathcal{O}_{U_{\sigma}}=\overline{\Theta^{\prime}}(\sigma)$. On each affine toric variety $U_{\sigma}$, we can take some $e_{j}$ such that $\chi_{j}^{e_{j}^{\vee}} \cdot \mathcal{O}_{U_{\sigma}}(E)=\mathcal{O}_{U_{\sigma}}$ as a subsheaf of the constant sheaf of the function field. We fix such $j$ and write $e_{\sigma}^{\vee}:=e_{j}^{\vee}$. The exact triangle,

$$
\overline{\Theta^{\prime}}(\sigma) \stackrel{\chi^{e_{\sigma}^{\vee}}}{\longrightarrow} \overline{\Theta^{\prime}}(\sigma) \longrightarrow \operatorname{Coker}\left(\chi^{e_{\sigma}^{\vee}}\right) \stackrel{[1]}{\longrightarrow} \overline{\Theta^{\prime}}(\sigma)[1]
$$

in $\mathrm{D}_{q c}^{\mathrm{b}}\left(M_{\mathbb{R}} / M\right)$ is sent to

$$
\bar{\Theta}(\sigma) \stackrel{\kappa_{\hat{\Sigma}}\left(\chi^{e_{\sigma}^{\vee}}\right)}{\longrightarrow} \bar{\Theta}(\sigma) \longrightarrow \kappa_{\hat{\Sigma}}\left(\operatorname{Coker}\left(\chi^{e_{\sigma}^{\vee}}\right)\right) \stackrel{[1]}{\longrightarrow} \bar{\Theta}(\sigma)[1]
$$

by $\kappa_{\hat{\Sigma}}$. 
On the other hand, there exists the following exact triangle in $\mathrm{D}_{q c}^{\mathrm{b}}\left(M_{\mathbb{R}} / M\right)$

$$
\mathbb{C}_{\sigma^{\vee}-e_{\sigma}^{\vee}} \longrightarrow \mathbb{C}_{\sigma^{\vee}} \stackrel{[1]}{\longrightarrow} \mathbb{C}_{Z_{\sigma}}[1] \longrightarrow \mathbb{C}_{\sigma^{\vee}}[1]
$$

where the left arrow is the restriction map and $Z_{\sigma}:=\left(\sigma^{\vee}-e_{\sigma}^{\vee}\right) \backslash\left(\sigma^{\vee}\right)$. Note that $Z_{\sigma}$ does not depend on the choice of $e_{\sigma}^{\vee}$. Applying $p_{\text {! }} \circ \mathbb{D}$ to this triangle, we have

$$
\bar{\Theta}(\sigma) \stackrel{\kappa_{\hat{\Sigma}}\left(\chi^{e_{\sigma}^{\vee}}\right.}{\longrightarrow} \bar{\Theta}(\sigma) \longrightarrow p_{!} \mathbb{D}\left(\mathbb{C}_{Z_{\sigma}}\right) \stackrel{[1]}{\longrightarrow} \bar{\Theta}(\sigma)[1] .
$$

Comparing (4.9) with (4.7), we have $\kappa_{\hat{\Sigma}}\left(\operatorname{Coker}\left(\chi^{e_{\sigma}^{\vee}}\right)\right) \cong p_{!} \mathbb{D}\left(\mathbb{C}_{Z_{\sigma}}\right)$.

We next calculate $\kappa_{\hat{\Sigma}}\left(\left[r_{i}\right]\right)$. For $\sigma \in \Sigma(i+1)$ and $\tau \in \Sigma(i)$, the restriction map $\mathcal{O}_{\sigma}(E) \rightarrow \mathcal{O}_{\tau}(E)$ is translated into $\overline{\Theta^{\prime}}(\sigma) \stackrel{\chi_{\tau}^{e_{\tau}^{\vee}}-e_{\sigma}^{\vee}}{\longrightarrow} \overline{\Theta^{\prime}}(\tau)$. We define the sheaf $\hat{\mathbb{C}}_{\sigma^{\vee}}:=\mathbb{C}_{\sigma^{\vee}-e_{\sigma}^{\vee}}$ on $M_{\mathbb{R}}$, then the restriction map $\hat{\mathbb{C}}_{\sigma^{\vee}} \rightarrow \hat{\mathbb{C}}_{\tau^{\vee}}$ is mapped to $\kappa_{\hat{\Sigma}}\left(\chi^{e_{\tau}^{\vee}-e_{\sigma}^{\vee}}\right)$ by applying $p_{!} \mathbb{D}$. On the other hand, we can see from the definition of $\kappa_{\Sigma}$ that the morphism induced on $\operatorname{Coker}\left(\epsilon_{i}\right) \rightarrow \operatorname{Coker}\left(\epsilon_{i-1}\right)$ is mapped to the composition of the restriction map $\mathbb{C}_{Z_{\sigma}} \rightarrow \mathbb{C}_{Z_{\tau}}$ and $p_{!} \mathbb{D}$. By summing up the restriction maps $\hat{\mathbb{C}}_{\sigma^{\vee}} \rightarrow \hat{\mathbb{C}}_{\tau^{\vee}}$ with Čech signs and applying $p_{!} \mathbb{D}$, we have $\kappa_{\hat{\Sigma}}\left(r_{i}\right)$. Hence, we conclude that $\kappa_{\hat{\Sigma}}\left(\left[r_{i}\right]\right)$ is obtained by summing up the restriction maps $\mathbb{C}_{Z_{\sigma}} \rightarrow \mathbb{C}_{Z_{\tau}}$ with Cech signs and applying $p_{!} \mathbb{D}$.

Note that $\hat{\mathbb{C}}_{\sigma}$ does not depend on a specific choice of $e_{\sigma}$. We can observe that $\left\{Z_{\sigma}\right\}_{\rho_{E} \subset \sigma \in \hat{\Sigma}(2)}$ forms a covering of $Z_{\rho_{E}} \backslash Z_{1}$. Moreover, $\left\{Z_{\sigma}\right\}_{\rho_{E} \subset \sigma \in \hat{\Sigma}}$ coincides with the Čech covering obtained from $\left\{Z_{\sigma}\right\}_{\rho_{E} \subset \sigma \in \hat{\Sigma}(2)}$. Hence, we have

$$
\mathbb{C}_{Z_{1}} \simeq\left(\bigoplus_{\sigma \in \hat{\Sigma}(1)} \mathbb{C}_{Z_{\sigma}} \rightarrow \cdots \rightarrow \bigoplus_{\sigma \in \hat{\Sigma}(n-1)} \mathbb{C}_{Z_{\sigma}} \rightarrow \bigoplus_{\sigma \in \hat{\Sigma}(n)} \mathbb{C}_{Z_{\sigma}}\right)
$$

where the differentials in the RHS are the Čech differentials, and we regard the first term in the RHS is in degree 0.

To sum up, we have

$$
\begin{aligned}
\mathbb{D} & \left(\mathbb{C}_{p\left(Z_{1}\right)}\right)[-n] \\
& \simeq p_{!} \mathbb{D}\left(\mathbb{C}_{Z_{1}}\right)[-n] \\
& \simeq p_{!} \mathbb{D}\left(\bigoplus_{\sigma \in \hat{\Sigma}(1)} \mathbb{C}_{Z_{\sigma}} \rightarrow \cdots \rightarrow \bigoplus_{\sigma \in \hat{\Sigma}(n-1)} \mathbb{C}_{Z_{\sigma}} \rightarrow \bigoplus_{\sigma \in \hat{\Sigma}(n)} \mathbb{C}_{Z_{\sigma}}\right)[-n]
\end{aligned}
$$




$$
\begin{aligned}
& \simeq \kappa_{\hat{\Sigma}}\left(\bigoplus_{\sigma \in \hat{\Sigma}(n)} \operatorname{Coker}\left(\chi^{e_{\sigma}^{\vee}}\right)[n] \rightarrow \bigoplus_{\sigma \in \hat{\Sigma}(n-1)} \operatorname{Coker}\left(\chi^{e_{\sigma}^{\vee}}\right)[n] \rightarrow \cdots\right. \\
& \left.\quad \rightarrow \bigoplus_{\sigma \in \hat{\Sigma}(1)} \operatorname{Coker}\left(\chi^{e_{\sigma}^{\vee}}\right)[n]\right)[-n] \\
& \simeq \kappa_{\hat{\Sigma}}\left(\mathcal{O}_{E}(E)\right) .
\end{aligned}
$$

By Theorem 3.3 (2) and (3), we have

$$
\begin{aligned}
\kappa_{\hat{\Sigma}}\left(\mathcal{O}_{E}(k E)\right) & \simeq \mathbb{D}\left(\mathbb{C}_{p\left(Z_{1}\right)}\right) \star \cdots \star \mathbb{D}\left(\mathbb{C}_{p\left(Z_{1}\right)}\right)[-n] \\
& \simeq \mathbb{D}\left(p_{*} \mathbb{C}_{Z_{k}}\right)[-n] .
\end{aligned}
$$

This completes the proof.

q.e.d.

Proof of Theorem 1.3. We note that $\kappa_{\hat{\Sigma}}\left(\mathcal{O}_{E}(k E)\right)$ is exceptional for $1 \leq$ $k \leq n-1$, since $\kappa_{\hat{\Sigma}}$ is fully-faithful and $\mathcal{O}_{E}(k E)$ is exceptional. We write the triangulated hull of $\mathcal{O}_{E}(k E)$ by $\mathrm{D}_{k}$. Then, $\kappa_{\hat{\Sigma}}\left(\mathrm{D}_{k}\right)$ is an admissible full subcategory of $\mathrm{D}_{c}^{\mathrm{b}}\left(M_{\mathbb{R}} / M, \overline{\Lambda_{\hat{\Sigma}}}\right)$. We have a semi-orthogonal decomposition [1],

$$
\begin{aligned}
& \mathrm{D}_{c}^{\mathrm{b}}\left(M_{\mathbb{R}} / M, \overline{\Lambda_{\hat{\Sigma}}}\right) \\
& \cong\left\langle\kappa_{\hat{\Sigma}}\left(\mathrm{D}_{-n+1}\right), \ldots, \kappa_{\hat{\Sigma}}\left(\mathrm{D}_{-1}\right),{ }^{\perp}\left\langle\kappa_{\hat{\Sigma}}\left(\mathrm{D}_{-n+1}\right), \ldots, \kappa_{\hat{\Sigma}}\left(\mathrm{D}_{-1}\right)\right\rangle\right\rangle .
\end{aligned}
$$

Hence, it is enough to show that $\perp\left\langle\kappa_{\hat{\Sigma}}\left(\mathrm{D}_{-n+1}\right), \ldots, \kappa_{\hat{\Sigma}}\left(\mathrm{D}_{-1}\right)\right\rangle=$ $\mathrm{D}_{c}^{\mathrm{b}}\left(M_{\mathbb{R}} / M, \overline{\Lambda_{\Sigma}}\right)$.

Step $1(\subset)$. For $\mathcal{E} \in \mathrm{D}_{c}^{\mathrm{b}}\left(M_{\mathbb{R}} / M, \overline{\Lambda_{\Sigma}}\right)$, we have

$$
\mathbb{R} \operatorname{Hom}\left(\mathcal{E}, \mathbb{D}\left(p_{*} \mathbb{C}_{Z_{k}}\right)\right) \simeq \mathbb{R} \operatorname{Hom}\left(p_{*} \mathbb{C}_{Z_{k}}, \mathbb{D}(\mathcal{E})\right) .
$$

We will show that this cohomology vanishes for $1 \leq k \leq n-1$.

Let us first reduce the vanishing of (4.14) to (2) of Lemma 4.2. We define

$$
F:=\left\{m=\sum_{i=1}^{n} a_{i} e_{i}^{\vee} \in M_{\mathbb{R}} \mid-1 \leq a_{i}<0\right\} \backslash M,
$$

and $\hat{Z}_{k}:=Z_{k} \cap F$, then

$$
=\bigsqcup_{\substack{1 \leq j \leq k \\
l_{1}+\cdots+l_{j}=-k \\
I:=\left\{i_{1}, \ldots, i_{j}\right\} \subset\{1, \ldots, n\}}}^{\bigsqcup_{k} \hat{Z}_{k}}\left\{m=\sum_{i=1}^{n} a_{i} e_{i}^{\vee} \in Z_{k} \mid \begin{array}{c}
l_{p} \leq a_{p}<l_{p}+1 \text { for any } p \in I, \\
-1 \leq a_{q}<0 \text { for any } q \in\{1, \ldots, k\} \backslash I
\end{array}\right\}
$$


By induction, we only have to show

$$
\mathbb{R H o m}\left(p_{*} \mathbb{C}_{\hat{Z}_{k}}, \mathbb{D}(\mathcal{E})\right) \simeq 0,
$$

to prove the vanishing of (4.14). Since there exists an exact triangle for $k \geq 2$

$$
\mathbb{C}_{\tilde{Z}_{k}} \rightarrow \mathbb{C}_{\hat{Z}_{k}} \rightarrow \mathbb{C}_{Z_{k-1}} \stackrel{[1]}{\rightarrow} \mathbb{C}_{\tilde{Z}_{k}}[1]
$$

where $\tilde{Z}_{k}:=\hat{Z}_{k}-\hat{Z}_{k-1}$, we can further reduce the vanishing to

$$
\mathbb{R H o m}\left(p_{*} \mathbb{C}_{\tilde{Z}_{k}}, \mathbb{D}(\mathcal{E})\right) \simeq \mathbb{R} \Gamma_{p\left(\tilde{Z}_{k}\right)}\left(M_{\mathbb{R}} / M, \mathbb{D} \mathcal{E}\right) \simeq 0,
$$

where we define $\tilde{Z}_{1}:=\hat{Z}_{1}$.

We define the subset $U_{k}$ of $M_{\mathbb{R}} / M$ by

$$
\begin{aligned}
& U_{k}:=p\left(\tilde{U}_{k}\right), \\
& \tilde{U}_{k}:=F \backslash \hat{Z}_{k-1},
\end{aligned}
$$

then $p\left(\tilde{Z}_{k}\right)$ is a closed subset of $U_{k}$ (Figures 3,4 and 5$)$. Here we set $\hat{Z}_{0}=\varnothing$. In the following, we fix an integer $k$ with $1 \leq k \leq n-1$. Then, we have the following exact triangle:

$$
\mathbb{R} \Gamma_{p\left(\tilde{Z}_{k}\right)}\left(U_{k}, \mathbb{D} \mathcal{E}\right) \rightarrow \mathbb{R} \Gamma\left(U_{k}, \mathbb{D} \mathcal{E}\right) \rightarrow \mathbb{R} \Gamma\left(U_{k+1}, \mathbb{D} \mathcal{E}\right) \stackrel{[1]}{\rightarrow} \mathbb{R}_{p\left(\tilde{Z}_{k}\right)}\left(U_{k}, \mathbb{D} \mathcal{E}\right)[1]
$$

Now the following lemma is clear:

Lemma 4.2. For $\mathcal{E} \in \mathrm{D}_{c}^{\mathrm{b}}\left(M_{\mathbb{R}} / M, \overline{\Lambda_{\hat{\Sigma}}}\right)$, the following are equivalent:

(1) $\mathbb{R H o m}\left(\mathcal{E}, \mathbb{D}\left(p_{*} \mathbb{C}_{Z_{k}}\right)\right) \simeq 0$ for $1 \leq k \leq n-1$.

(2) $\mathbb{R} \Gamma\left(U_{k}, \mathbb{D} \mathcal{E}\right) \stackrel{\simeq}{\rightarrow} \mathbb{R} \Gamma\left(U_{k+1}, \mathbb{D} \mathcal{E}\right)$ for $1 \leq k \leq n-1$.

We will show the isomorphism (2) above in what follows. Let us define the family of open subsets $\mathcal{V}:=\left\{V_{s}\right\}_{(-\infty, 1)}$ of $U_{k}$ (Figures 6 and 7) by

$$
V_{s}:= \begin{cases}U_{k+1} & \text { for } s \in(-\infty, 0), \\ U_{k+1} \cup p\left(\left(\tilde{U}_{k}-\frac{1-s}{n} \cdot e_{E}\right) \cap F\right) & \text { for } s \in[0,1) .\end{cases}
$$

Then, $\mathcal{V}$ obviously satisfies (1) of Theorem 2.3 .

For $s \leq t<1$, we have

$$
V_{t} \backslash V_{s}=\left\{\begin{array}{l}
\varnothing \text { or } \\
p\left(\left(\tilde{U}_{k}-\frac{1-s}{n} \cdot e_{E}\right) \cap\left\{n \in N_{\mathbb{R}} \mid\left\langle n, e_{E}\right\rangle \geq-k-t\right\} \cap F\right) .
\end{array}\right.
$$

It follows that $V_{t} \backslash V_{s}$ is relatively compact in $U_{k}$, i.e., $\mathcal{V}$ satisfies (2) of Theorem 2.3. 

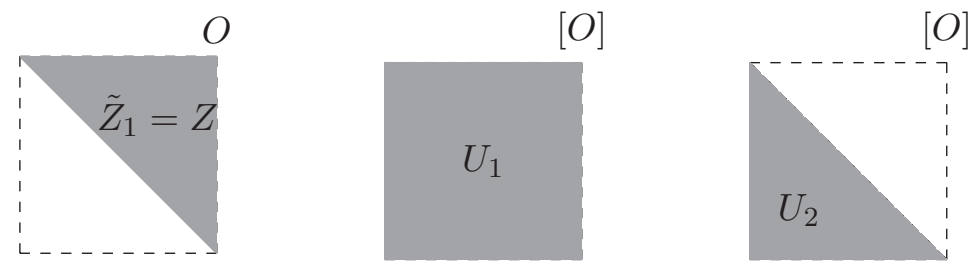

Figure 3. $\tilde{Z}_{1}, U_{1}$, and $U_{2}$ in $n=2$.
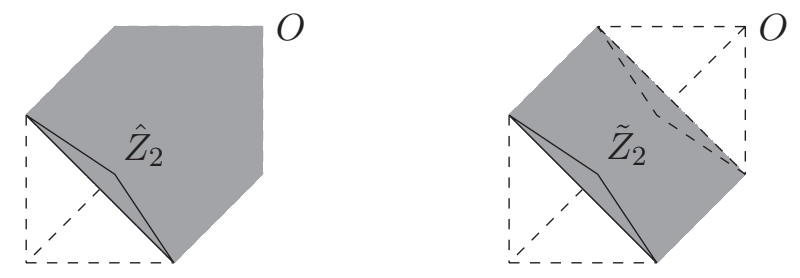

Figure 4. $\hat{Z}_{2}$ and $\tilde{Z}_{2}$ in $n=3$.
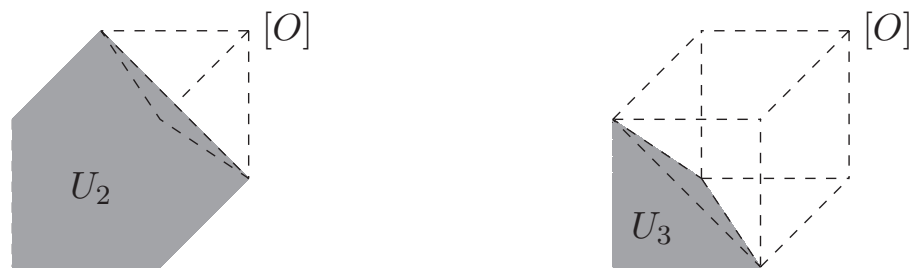

Figure 5. $U_{2}$ and $U_{3}$ in $n=3$.

For $s \leq t$, we have

$$
\bigcap_{u>s} \operatorname{Cl}\left(V_{u} \backslash V_{s}\right) \backslash V_{t}= \begin{cases}p\left(\left(\partial \tilde{U}_{k}-\frac{1-s}{n} \cdot e_{E}\right) \cap F\right) & \text { if } s=t>0 \\ \varnothing & \text { otherwise. }\end{cases}
$$

Take $y \in p\left(\left(\partial \tilde{U}_{k}-\frac{1-s}{n} \cdot e_{E}\right) \cap F\right)$. We can see that there exists a neighborhood $B(y)$ of $y$ such that $\gamma:=N_{y}\left(U_{k} \backslash V_{s}\right)$ and $U_{k} \backslash V_{s}$ is canonically isomorphic in $B(y)$. The cone $\gamma$ is an $n$-dimensional closed polyhedral convex cone which is contained in $e_{E}^{\vee}$ under the canonical identification $T_{y} M_{\mathbb{R}} / M \cong M_{\mathbb{R}}$. Take an $n$-dimensional proper closed polyhedral covex cone $\delta \subset N_{\mathbb{R}}$ such that $\sum_{i=1}^{n} \mathbb{R}_{\geq 0} \cdot e_{i} \backslash\{0\} \subset \operatorname{Int}(\delta)$ and $\delta \subset \operatorname{Int}\left(e_{E}^{\vee}\right)$. We also have $\gamma \subset e_{E}^{\vee}$. Hence we have $-\delta \cap \gamma=\{0\}$. We can also see 

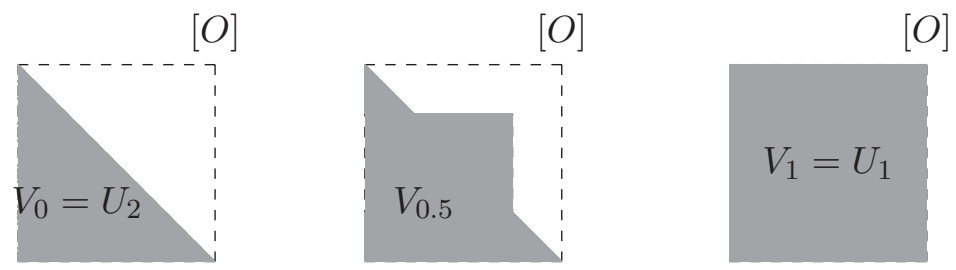

Figure 6. $V_{0}, V_{0.5}$, and $V_{1}$ in $n=2$.
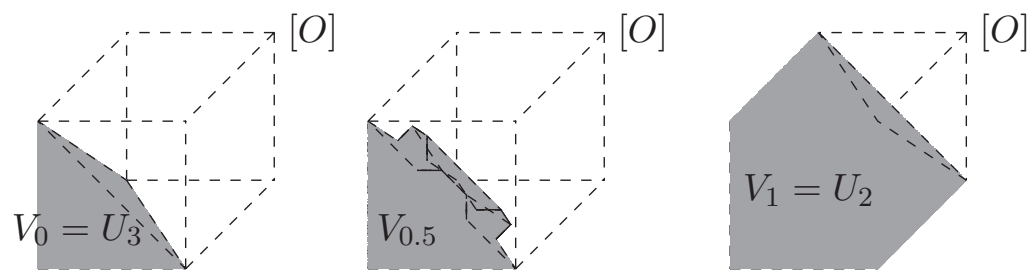

Figure 7. $V_{0}, V_{0.5}$ and $V_{1}$ in $n=3$.

that $\delta^{\vee} \backslash\{0\} \subset \sum_{i=1}^{n} \mathbb{R}_{>0} \cdot e_{i}^{\vee}$ and

$$
\begin{aligned}
\gamma^{\vee}=N_{y}^{*}\left(U_{k} \backslash V_{s}\right) & \subset N_{y}^{*}\left(p\left(\tilde{U}_{k}-\frac{1-s}{n} \cdot e_{E}\right)\right)+N_{y}^{*}(p(F)) \\
& \subset \sum_{i=1}^{n} \mathbb{R}_{\geq 0} \cdot e_{i} .
\end{aligned}
$$

Hence we have Relint $\left(\gamma^{\vee}\right)+\delta^{\vee} \subset \sum_{i=1}^{n} \mathbb{R}_{>0} \cdot e_{i}$.

Since $\operatorname{SS}(\mathbb{D} \mathcal{E})=-\operatorname{SS}(\mathcal{E}) \subset-\bar{\Lambda}_{\Sigma}$, we have $U_{k} \times\left(\bigcup_{i=1}^{n} \mathbb{R}_{>0} e_{i}\right) \cap$ $\mathrm{SS}(\mathbb{D} \mathcal{E})=\varnothing$. Hence, by Lemma 2.7, we obtain $\left(\mathbb{R} \Gamma_{U_{k} \backslash V_{s}}(\mathbb{D} \mathcal{E})\right)_{y} \simeq 0$. Then we can use Theorem 2.3 for $\mathcal{V}$ and we know the middle arrow of (4.22) is an isomorphism. By Lemma 4.2, we conclude that $\mathcal{E} \in \in^{\perp}\left\langle\kappa_{\hat{\Sigma}}\left(\mathrm{D}_{-n+1}\right), \ldots, \kappa_{\hat{\Sigma}}\left(\mathrm{D}_{-1}\right)\right\rangle$.

Step $2(\supset)$. Conversely, take $\mathcal{E} \in{ }^{\perp}\left\langle\kappa_{\hat{\Sigma}}\left(\mathrm{D}_{-r+1}\right), \ldots, \kappa_{\hat{\Sigma}}\left(\mathrm{D}_{-1}\right)\right\rangle$. We have to show that $\mathrm{SS}(\mathcal{E}) \subset \overline{\Lambda_{\Sigma}}$, or equivalently,

$$
\operatorname{SS}(\mathcal{E}) \cap \bigcup_{\substack{\rho_{E} \subset \sigma \in \hat{\Sigma} \\ \operatorname{dim} \sigma<n}} p\left(\operatorname{Relint}\left(\left(\sigma^{\perp}+M\right) \cap F\right)\right) \times(-\sigma)=\varnothing .
$$

For a proof by contradiction, we assume that there exists an element $\left(\left[x_{0}\right],-\xi_{0}\right)$ in the LHS of the above $(4.27)$. Since $\operatorname{SS}(\mathcal{E})$ is a conic Lagrangian subset $[6]$, we can assume that $\xi_{0} \in \operatorname{Relint}(\sigma)$ for some $\sigma \in \hat{\Sigma}$ such that $\rho_{E} \subset \sigma$ and $\operatorname{dim} \sigma<n$. Fix $k \in\{1, . ., n-1\}$, then we can take the unique lift $x_{0}=\sum_{i=1}^{n} a_{i} e_{i}^{\vee} \in M_{\mathbb{R}}$ satisfying $-1 \leq a_{i}<0$, $\sum_{i=1}^{n} a_{i}=-k$. 
We can see that there exists a neighborhood $B\left(\left[x_{0}\right]\right)$ of $\left[x_{0}\right]$ such that $\tilde{\gamma}:=N_{\left[x_{0}\right]}\left(U_{k} \backslash U_{k+1}\right)$ is canonically isomorphic to $U_{k} \backslash U_{k+1}$ in $B\left(\left[x_{0}\right]\right)$. Moreover, via the canonical identification $T_{\left[x_{0}\right]} M_{\mathbb{R}} / M \cong M_{\mathbb{R}}$, we have $\tilde{\gamma} \cong \sigma^{\vee}$. On the other hand, we can take an $n$-dimensional proper polyhedral closed convex cone $\tilde{\delta}$ such that $\sum_{i=1}^{n} \mathbb{R}_{\geq 0} \cdot e_{i}^{\vee} \backslash\{0\} \subset \operatorname{Int}(\tilde{\delta})$, $\tilde{\delta}^{\vee} \cap\left(\tilde{\gamma}^{\vee} \backslash \operatorname{Relint}\left(\tilde{\gamma}^{\vee}\right)\right)=\{0\}$, and $\xi_{0} \in \operatorname{Int}\left(\tilde{\delta}^{\vee}\right)$.

The cones $\tilde{\gamma}$ and $\tilde{\delta}$ clearly satisfy (ii)-(iv) of Lemma 2.8. By the definitions of $\tilde{\gamma}$ and $\tilde{\delta},\left(\tilde{\delta}^{\vee}+\tilde{\gamma}^{\vee}\right) \subset \sum_{i=1}^{n} \mathbb{R}_{\geq 0} \cdot e_{i}^{\vee}$. Since $\operatorname{SS}(\mathcal{E}) \subset \overline{\Lambda_{\hat{\Sigma}}}$, we have

$$
\begin{aligned}
\operatorname{SS}(\mathbb{D} \mathcal{E}) & \cap\left(B\left(\left[x_{0}\right]\right) \backslash \tilde{\gamma}\right) \times\left(\tilde{\delta}^{\vee}+\tilde{\gamma}^{\vee}\right) \\
& \subset-\overline{\Lambda_{\hat{\Sigma}}} \cap\left(B\left(\left[x_{0}\right]\right) \backslash \tilde{\gamma}\right) \times\left(\sum_{i=1}^{n} \mathbb{R}_{\geq 0} e_{i}\right) \\
& \subset \bigcup_{0 \neq \tau \prec \sigma} \tau^{\perp} \times \tau \cup\left(B\left(\left[x_{0}\right]\right) \backslash \tilde{\gamma}\right) \times\{0\} \\
= & \bigsqcup_{0 \neq \tau \prec \sigma=\tilde{\gamma}^{\vee}} R_{\tau} \times \tau \cup\left(B\left(\left[x_{0}\right]\right) \backslash \tilde{\gamma}\right) \times\{0\} .
\end{aligned}
$$

In the second line, we use the canonical identification $B\left(\left[x_{0}\right]\right) \hookrightarrow$ $T_{\left[x_{0}\right]} M_{\mathbb{R}} / M \cong M_{\mathbb{R}}$. This shows that $\tilde{\delta}$ and $\tilde{\gamma}$ also satisfy (i) of Lemma 2.8.

By Lemma 2.8, there exists $x$ near $x_{0}$ such that

$$
\mathbb{R} \Gamma_{U_{k} \backslash U_{k+1}}\left(p\left((x-\tilde{\delta}) \cap \tilde{U}_{k} \backslash \tilde{U}_{k+1}\right), \mathbb{D} \mathcal{E}\right) \not 0 .
$$

We define $S:=p\left(\tilde{U}_{k} \backslash \tilde{U}_{k+1} \cap(x-\tilde{\delta})\right)$. Then we have

$$
\mathbb{R} \Gamma\left(U_{k+1} \cup S, \mathbb{D} \mathcal{E}\right) \nsucceq \mathbb{R} \Gamma\left(U_{k+1}, \mathbb{D} \mathcal{E}\right) .
$$
by

We define a family of open subsets $\mathcal{V}^{\prime}:=\left\{V_{s}^{\prime}\right\}_{s \in(-\infty, 1)}$ in $U_{k}$ (Figure 8)

$$
V_{s}^{\prime}:= \begin{cases}U_{k+1} \cup S & \text { for } s \in(-\infty, 0), \\ U_{k+1} \cup S \cup p\left(F \cap\left(s \cdot\left(\tilde{U}_{k}-x\right)+x\right)\right) & \text { for } s \in[0,1) .\end{cases}
$$

We can see that the family $\mathcal{V}^{\prime}$ satisfies (1) and (2) of Theorem 2.3.

For $s \leq t$, we have

$$
\bigcap_{u>s} \mathrm{Cl}\left(V_{u}^{\prime} \backslash V_{s}^{\prime}\right) \backslash V_{t}^{\prime}= \begin{cases}W_{1} \cup W_{2} \cup W_{3} & s=t \geq 0, \\ \varnothing & \text { otherwise, }\end{cases}
$$




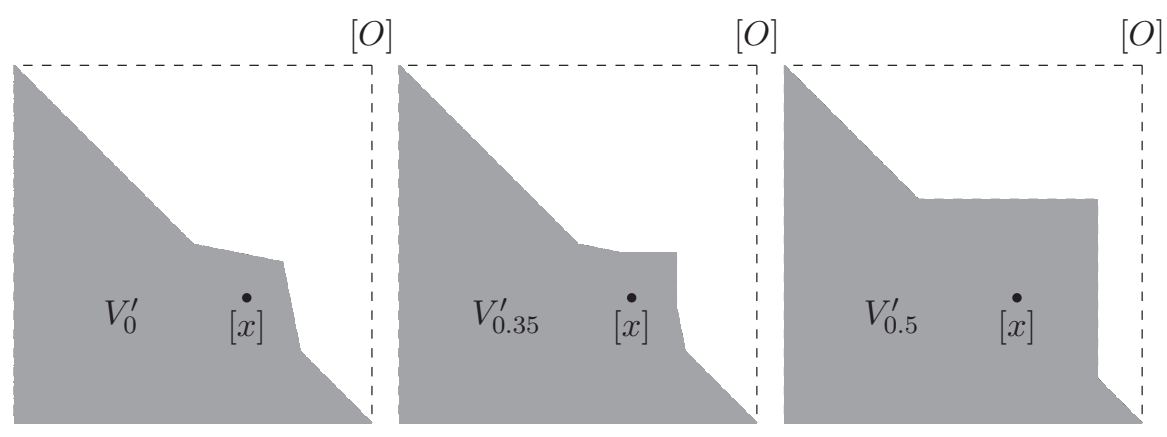

Figure 8. $V_{0}^{\prime}, V_{0.35}^{\prime}$, and $V_{0.5}^{\prime}$ in $n=2$.

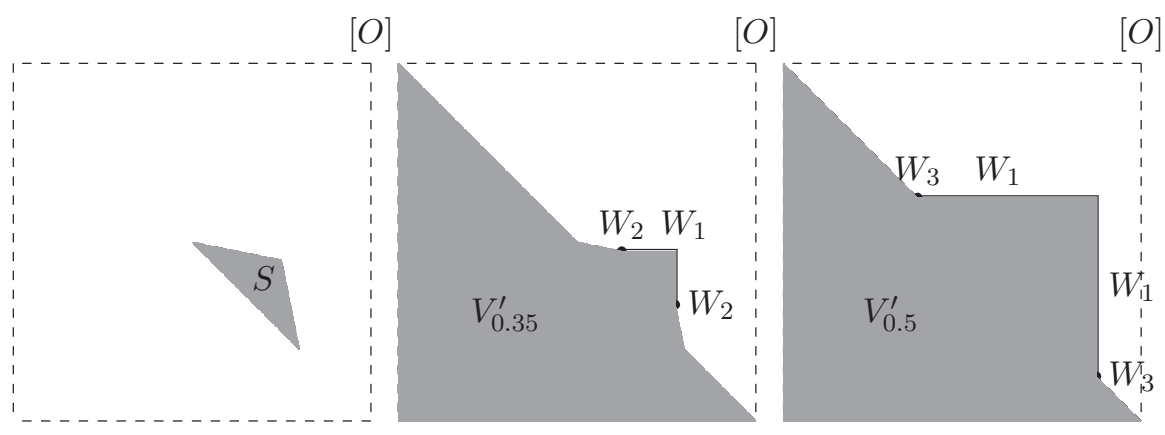

Figure 9. $S, W_{i}$ in $n=2$.

where

$$
\begin{aligned}
& W_{1}:=p\left(F \cap\left(s \cdot\left(\partial \tilde{U}_{k}-x\right)+x\right)\right) \backslash\left(\mathrm{Cl}\left(U_{k+1}\right) \cup S\right), \\
& W_{2}:=\partial S \cap p\left(F \cap\left(s \cdot\left(\partial \tilde{U}_{k}-x\right)+x\right)\right) \backslash W_{3}, \\
& W_{3}:=p\left(\left\{m \in M_{\mathbb{R}} \mid\left\langle m, e_{E}\right\rangle=-k-1\right\} \cap\left(s \cdot\left(\partial \tilde{U}_{k}-x\right)+x\right)\right) .
\end{aligned}
$$

Some examples of $W_{i}$ are depicted in Figure 9.

We take $y \in \bigcap_{u>s} \mathrm{Cl}\left(V_{u}^{\prime} \backslash V_{s}^{\prime}\right) \backslash V_{s}^{\prime}$ for $0 \leq s<1$ and define a cone $\hat{\gamma}$ as $\hat{\gamma}:=N_{y}\left(U_{k} \backslash V_{s}^{\prime}\right)$. There exists a neighborhood $B\left(y^{\prime}\right)$ of $y^{\prime}$ such that $\gamma$ is canonically isomorphic to $U_{k} \backslash V_{s}^{\prime}$ in $B\left(y^{\prime}\right)$.

If $y \in W_{2}$, we have $\hat{\gamma}^{\vee} \subset \sum_{i=1}^{n} \mathbb{R}_{\geq 0} \cdot e_{i}$ via the canonical identification $T_{y}^{*} M_{\mathbb{R}} / M \cong N_{\mathbb{R}}$. Since $\mathbb{D} \mathcal{E}$ does not have its microsupport in its first quadrant at $y$, we have $\left(\mathbb{R} \Gamma_{U_{k} \backslash V_{s}^{\prime}}(\mathbb{D} \mathcal{E})\right)_{y} \simeq 0$ by Lemma 2.6.

If $y \in W_{1}$, we have $\hat{\gamma}^{\vee} \subset \sum_{i=0} \mathbb{R}_{\geq 0} \cdot e_{i}$. On the other hand, the microsupport of $\mathbb{D} \mathcal{E}$ at $y$ is contained in a face of $\sum_{i=1}^{n} \mathbb{R}_{\geq 0} \cdot e_{i}$. Hence we have $\left(\mathbb{R} \Gamma_{U_{k} \backslash V_{s}^{\prime}}(\mathbb{D} \mathcal{E})\right)_{y} \simeq 0$ by Lemma 2.7 . 
If $y \in W_{3}, \hat{\gamma}$ is contained in some $\sigma \in \hat{\Sigma}$ such that $\rho_{E} \subset \sigma$. Hence, again by Lemma 2.7 , we have $\left(\mathbb{R} \Gamma_{U_{k} \backslash V_{s}^{\prime}}(\mathbb{D} \mathcal{E})\right)_{y} \simeq 0$.

Then, by using Theorem 2.3 for $\mathcal{V}^{\prime}$, we have

$$
\mathbb{R} \Gamma\left(U_{k}, \mathbb{D} \mathcal{E}\right) \simeq \mathbb{R} \Gamma\left(U_{k+1} \cup S, \mathbb{D} \mathcal{E}\right) .
$$

As a consequence, we have the following commutative diagram:

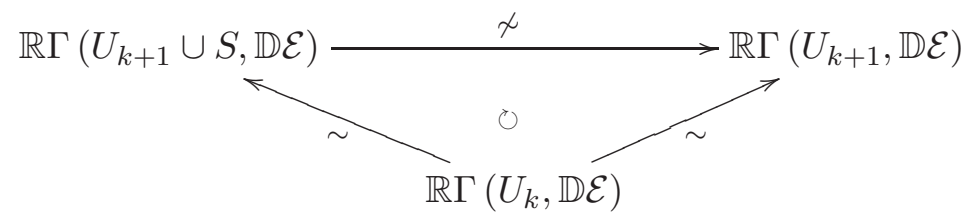

Since all arrows in (4.35) are restriction morphisms, this diagram is commutative. The upper arrow is (4.30) and the right arrow is the isomorphism (4.34). The left arrow is also an isomorphism by the assumption and Lemma 4.2. This diagram is obviously absurd. Hence, $\left(\left[x_{0}\right], \xi_{0}\right) \notin \mathrm{SS}(\mathcal{E})$. This completes the proof. q.e.d.

\section{Proofs of the main theorems}

Proof of Theorem 1.4. First, suppose that $\kappa_{\hat{\Sigma}}: \mathrm{D}^{\mathrm{b}}\left(\operatorname{coh} X_{\hat{\Sigma}}\right) \rightarrow$ $\mathrm{D}_{c}^{\mathrm{b}}\left(M_{\mathbb{R}} / M, \overline{\Lambda_{\hat{\Sigma}}}\right)$ is an equivalence. Then, semi-orthogonal decomposition described in Orlov's theorem (1.3) implies

$$
{ }^{\perp}\left\langle\kappa_{\hat{\Sigma}}\left(\mathcal{O}_{E}(k E)\right)\right\rangle_{1 \leq k \leq n-1} \cong \kappa_{\hat{\Sigma}}\left(\pi^{*} \mathrm{D}^{\mathrm{b}}\left(\operatorname{coh} X_{\Sigma}\right)\right) .
$$

On the other hand, Theorem 1.3 implies

$$
{ }^{\perp}\left\langle\kappa_{\hat{\Sigma}}\left(\mathcal{O}_{E}(k E)\right)\right\rangle_{1 \leq k \leq n-1} \cong \iota\left(\mathrm{D}_{c}^{\mathrm{b}}\left(M_{\mathbb{R}} / M, \overline{\Lambda_{\hat{\Sigma}}}\right)\right) .
$$

Hence,

$$
\begin{aligned}
\kappa_{\Sigma}\left(\mathrm{D}^{\mathrm{b}}\left(\operatorname{coh} X_{\Sigma}\right)\right) & \cong \kappa_{\hat{\Sigma}}\left(\pi^{*} \mathrm{D}^{\mathrm{b}}\left(\operatorname{coh} X_{\Sigma}\right)\right) \cong \perp\left\langle\kappa_{\hat{\Sigma}}\left(\mathcal{O}_{E}(k E)\right)\right\rangle_{1 \leq k \leq n-1} \\
& \cong \iota\left(\mathrm{D}_{c}^{\mathrm{b}}\left(M_{\mathbb{R}} / M, \overline{\Lambda_{\Sigma}}\right)\right) \cong \mathrm{D}_{c}^{\mathrm{b}}\left(M_{\mathbb{R}} / M, \overline{\Lambda_{\Sigma}}\right),
\end{aligned}
$$

where the equivalence in the first line is Theorem 3.3 (1).

Conversely, we assume that Conjecture 1.1 holds for $\Sigma$. Take $\mathcal{E} \in$ $\kappa_{\hat{\Sigma}}\left(\mathrm{D}^{\mathrm{b}}\left(\operatorname{coh} X_{\hat{\Sigma}}\right)\right)^{\perp}$, then $\mathcal{E} \in \kappa_{\hat{\Sigma}}\left(\mathcal{O}_{E}(k E)\right)^{\perp}$ for $1 \leq k \leq n-1$ and $\mathcal{E} \in \kappa_{\hat{\Sigma}}\left(\pi^{*} \mathrm{D}^{\mathrm{b}} \operatorname{coh}\left(X_{\Sigma}\right)\right) \cong \iota\left(\mathrm{D}^{\mathrm{b}}\left(M_{\mathbb{R}} / M, \overline{\Lambda_{\Sigma}}\right)\right)^{\perp}$. Hence, by Theorem 1.3, $\mathcal{E} \cong 0$. This completes the proof.

q.e.d.

Using Theorem 1.4, we have a proof of Conjecture 1.1 for smooth complete toric surfaces.

Proof of Theorem 1.2. We use toric minimal model program for toric surfaces. For any toric surfaces, after some toric blow-downs, we have 
$\mathbb{P}^{2}, \mathbb{P}^{1} \times \mathbb{P}^{1}$ or Hirzebruch surfaces $\mathbb{F}_{n}$. By Theorem 1.4, it is enough to show that Conjecture 1.1 holds for these toric minimal models. Moreover, $\mathbb{P}^{2}$ and $\mathbb{F}_{n}$ are obtained by successive blow-ups and blow-downs of $\mathbb{P}^{1} \times \mathbb{P}^{1}$. Hence, all cases may be reduced to the Conjecture 1.1 for $\mathbb{P}^{1} \times \mathbb{P}^{1}$. The last case has already been shown by Treumann (Theorem $3.2(1))$. This completes the proof.

q.e.d.

Finally, we obtain Corollary 1.5 by combining Theorem 1.2 with Theorem 3.1.

\section{References}

[1] A. I. Bondal and M. M. Kapranov, Representable functors, Serre functors, and reconstructions, Izv. Akad. Nauk SSSR Ser. Mat. 53 (1989), no. 6, 1183-1205, 1337, MR1039961, Zbl 0703.14011

[2] A. I. Bondal and D. Orlov, Derived categories of coherent sheaves, Proceedings of the International Congress of Mathematicians, Vol. II (Beijing, 2002), 2002, pp. 47-56, MR1957019, Zbl 0996.18007

[3] A. I. Bondal, Derived categories of toric varieties, Convex and Algebraic geometry, Oberwolfach conference reports 3 (2006) 284-286.

[4] B. Fang, C.-C. M. Liu, D. Treumann, and E. Zaslow, A categorification of Morelli's theorem, Invent. Math.186 (2011), no. 1, 79-114, MR2836052, Zbl 1250.14011

[5] B. Fang, C.-C. M. Liu, D. Treumann, and E. Zaslow, T-duality and homological mirror symmetry for toric varieties, Adv. Math. 229 (2012), no. 3, 1875-1911, MR2871160, Zbl 1260.14049

[6] M. Kashiwara and P. Schapira. (1990). Sheaves on manifolds, Grundlehren der Mathematischen Wissenschaften [Fundamental Principles of Mathematical Sciences], vol. 292, Springer-Verlag, Berlin, ISBN 3-540-51861-4. With a chapter in French by Christian Houzel, MR1074006, Zbl 0709.18001

[7] D. Nadler, Microlocal branes are constructible sheaves, Selecta Math. (N.S.) 15 (2009), no. 4, 563-619, MR2565051, Zbl 1197.53116

[8] D. Nadler and E. Zaslow, Constructible sheaves and the Fukaya category, J. Amer. Math. Soc. 22 (2009), no. 1, 233-286, MR2449059, Zbl 1227.32019

[9] D. O. Orlov, Projective bundles, monoidal transformations, and derived categories of coherent sheaves, Izv. Ross. Akad. Nauk Ser. Mat. 56 (1992), no. 4, 852-862, MR1208153, Zbl 0798.14007

[10] S. Scherotzke and N. Sibilla, The non-equivariant coherent-constructible correspondence and a conjecture of King, Selecta Math. (N.S.) 22 (2016), no. 1, 389-416. MR3437841, Zbl 06537958

[11] D. Treumann, Remarks on the nonequivariant coherent-constructible correspondence for toric varieties, eprint arXiv:1006.5756 (2010).

Graduate School of Mathematical Sciences

UNIVERSITY OF TOKYO

Komaba Meguroku, Tokyo 153-8914

JAPAN

E-mail address: kuwagaki@ms.u-tokyo.ac.jp 